\title{
The Theory of Everything as the Reversibility Theory
}

\author{
Ding-Yu Chung \\ Utica, MI, USA \\ Email: dy chung@yahoo.com
}

Received 31 August 2015; accepted 19 October 2015; published 23 October 2015

Copyright (C 2015 by author and Scientific Research Publishing Inc.

This work is licensed under the Creative Commons Attribution International License (CC BY). http://creativecommons.org/licenses/by/4.0/

(c) (i) Open Access

\section{Abstract}

In the posited reversibility theory, 1) all physical laws and phenomena are permanently reversible, 2) all physical laws are derived from the reversible M-theory (extension of string theory) in the reversible multiverse, and 3) temporary irreversible entropy increase is allowed through reversibility breaking, symmetry breaking, and low entropy beginning. Dealing with all physical laws and phenomena, the reversibility theory is the theory of everything, including thermodynamic, cosmology, the composition (baryonic matter, dark matter, and dark energy) in the universe, the periodic table of all elementary particles, the galaxy evolution, superconductivity, and black hole. In the reversible M-theory, space-time dimension number oscillates between 11D (space-time dimension) and 10D and between 10D and 4D. The reversible M-theory includes 11D membrane, 10D string, and variable $D$ particle. Space-time dimension number between 10 and 4 decreases with decreasing speed of light, decreasing vacuum energy, and increasing rest mass. In our dual universe of positive-negative energy universe, the reversible oscillation between 10D with and 4D in the negative energy universe without kinetic energy is the reversible cyclic fractionalization-condensation for the reversible cyclic expansion-contraction of the universe. Without kinetic energy, the individual particles have no individual momenta for entropy increase. The negative energy universe is the locally reversible universe for dark energy. In the positive energy universe where we live, the absorption of the interuniversal void forced the direct transformation from 10D to 4D as the inflation followed by the Big Bang, and created kinetic energy that provided individual momenta for individual particles to produce irreversible entropy increase, resulting in the reversibility breaking of the reversible oscillation. The positive energy universe is the locally irreversible universe. The dual universe as a whole is reversible guided by the reversible negative energy universe. As a result, the irreversible positive energy universe with the irreversible entropy increase is temporary, and will disappear.

\section{Keywords}

The Theory of Everything, Reversibility Theory, Entropy, Thermodynamic, Cyclic Universe, 


\section{Introduction}

In the second law of thermodynamics, the entropy (a measure of the disorder of a system) of an isolated system can increase, but not decrease. In other words, the entropy of a closed system will never decrease into the future. There are two mysteries about this irreversible entropy increase as described in "From Eternity to Here: The Quest for the Ultimate Theory of Time” by Sean Carroll [1]. Firstly, this irreversible entropy increase of a macroscopic collection of particles is different from all microscopic reversible processes where for every allowed process there exists a time-reversed process that is also allowed. Secondly, the universe started with the very low entropy state as the inflation-Big Bang in a very small space, not with the high entropy state near equilibrium state in a large space. Such low entropy beginning is a mystery. In this paper, the mysteries of the irreversible entropy increase are explained by the posited reversibility theory.

In the posited reversibility theory, 1) all physical laws and phenomena are permanently reversible, 2) all physical laws are derived from the reversible M-theory(extension of string theory) in the reversible multiverse, and 3) temporary irreversibility of entropy increase is allowed through reversibility breaking, symmetry breaking, and low entropy beginning as described in the previous papers [2] [3]. Dealing with all physical laws and phenomena, the reversibility theory is the theory of everything, including 1) thermodynamic in this paper, 2) cosmology [2]-[4], 3) the composition (baryonic matter, dark matter, and dark energy) in the universe [5] [6], 4) the periodic table of elementary particles [7]-[9] for all elementary particles (quarks, leptons, gauge bosons, the Higgs boson, and the knees-ankles-toe in cosmic rays), 5) the galaxy evolution [10] [11], 6) superconductivity [12], and 7) black hole [13] [14]. The theoretical results are in good agreement with the observations, and the calculated values from cosmology, the composition in the universe, and the periodic table of elementary particles are in good agreement with the observed values. This paper deals with thermodynamic of the reversibility theory.

In the reversible M-theory [6], space-time dimension number oscillates between 11D (space-time dimension) and 10D and between 10D and 4D without the conventional compactization of string. The reversible M-theory includes 11D membrane, 10D string, and variable $\mathrm{D}$ particle from $4 \mathrm{D}$ to $11 \mathrm{D}$. Space-time dimension number between 10 and 4 decreases with decreasing speed of light, decreasing vacuum energy, and increasing rest mass. 4D has zero vacuum energy. The reversibility theory posits that the multiverse is reversible, so all universes in the multiverse are reversible cyclic universes which have the inexhaustible resources of space-time to expand. In the reversible multiverse, one of the most important exclusions is the exclusion of the collision of expanding universes which cannot be reversed. The exclusion of the collision of expanding universes leads to the formation of the "interuniversal voids" that is functioned as the permanently gap among universes to keep universes apart. To prevent the collision, the interuniversal void detaches the incoming mass-energy in the interuniversal void to keep expanding universes apart without collision. When our observable universe absorbed the interuniversal void, the absorbed interuniversal void with the property of the detachment of mass-energy was transformed into the reverse Higgs field (the massless particle generating field) that detached adjacent mass-energy in the universe, resulting in the conversion of rest mass (massive particles) into kinetic energy (massless particles) starting the Big Bang. The absorption of the interuniversal void with zero vacuum energy also started the inflation by converting the 10D universe with high vacuum energy universe into the 4D universe with zero vacuum energy universe. The inflation followed by the Big Bang occurred in our observable universe [3].

A zero-sum energy dual universe of positive energy universe and negative energy universe can be created in the interuniversal void, and the new dual universe is again surrounded by the interuniversal void. Under symmetry, the new positive and negative energy universes undergo mutual annihilation to reverse to the interuniversal void immediately. Under symmetry breaking as in our dual universe, the new positive energy universe absorbs the interuniversal void, while the new negative energy does not. In our dual universe, the reversible oscillation between 10D with and 4D in the negative energy universe without kinetic energy is the reversible cyclic fractionalization-condensation for the cyclic expansion-contraction of the universe. Without kinetic energy, the individual particles have no individual momenta for entropy increase. As a result, the negative energy universe is the locally reversible universe ass dark energy. In the positive energy universe where we live, the absorption of 
the interuniversal void forced the direct transformation from 10D to $4 \mathrm{D}$ as the inflation followed by the Big Bang, and created kinetic energy that provided individual momenta for individual particles to produce irreversible entropy increase in a macroscopic collection of particles, resulting in the reversibility breaking of the reversible oscillation between 10D and 4D. As a result, the positive energy universe is the locally irreversible universe. Guided by the reversible negative energy universe the dual universe as a whole is reversible involving the reversible absorption-desorption of the interuniversal void. Consequently, the irreversible positive energy universe with the irreversible entropy increase is temporary, and will disappear.

The explanation of the first mystery of the irreversible entropy increase is that all physical laws are permanently reversible, and the temporary reversible entropy increase derived from the absorption of the interuniversal void will disappear. The explanation of the second mystery of the irreversible entropy increase is that to have exactly reversible absorption-desorption of the interuniversal void for the reversible dual universe, the absorption and desorption have to be uniform. The space of the universe, such as the 10D universe, where the absorption-desorption occurs has to be small enough for the uniform absorption-desorption. A small space results in a low entropy beginning such as the inflation-Big Bang for the beginning of the irreversible entropy increase.

The purpose of the paper is to explain the reversibility theory as the theory of everything including all physical laws and phenomena [2]-[14] derived from the reversible M-theory in the reversible multiverse described previously [2] [3] and irreversible entropy increase described in this paper. Section 2 describes the reversible M-theory based on space-time number oscillation. Section 3 explains the reversible multiverse. The reversible cyclic dual universe with irreversibility breaking and symmetry breaking, and low entropy beginning is explained in Section 4. Section 5 explains temporary irreversible entropy increase.

\section{The Reversible M-Theory}

In the posited reversibility theory, all physical laws and phenomena are permanently reversible, and all physical laws are derived from the reversible M-theoryas described previously [6]. M-theory requires the presences of 10D (space-time dimension) and 11D for string and membrane, respectively, in contrast to the observed 4D. In conventional M-theory with fixed space-time dimension number, the explanation of the hidden extra space dimensions is the compactization of the extra space dimensions, so space-time appears to be $4 \mathrm{D}$. In the reversible M-theory, dimension number oscillates between 11D and 10D and between 10D and 4D dimension by dimension reversibly. There is no compactization. The objects in the reversible M-theory includes $11 \mathrm{D}$ membrane $\left(2_{11}\right)$ as membrane (denoted as 2 for 2 space dimensions) in 11D, 10D string $\left(1_{10}\right)$ as string (denoted as 1 for 1 space dimension) in $10 \mathrm{D}$, and variable $\mathrm{D}$ particle $\left(0_{4-11}\right)$ as particle (denoted as 0 for 0 space dimension) in $4 \mathrm{D}$ to $11 \mathrm{D}$.

Space-time dimension number varies with varying speed of light, vacuum energy, and rest mass. Varying speed of light has been proposed to explain the horizon problem of cosmology [15]. J. D. Barrow [16] proposes that the time dependent speed of light varies as some power of the expansion scale factor $a$ in such way that

$$
c(t)=c_{0} a^{n},
$$

where $\mathrm{c}_{0}>0$ and $n$ are constants. The increase of speed of light is continuous.

In this paper, varying dimension number (VDN) relates to quantized varying speed of light (QVSL), where the speed of light is invariant in a constant space-time dimension number, and the speed of light varies with varying space-time dimension number from 4 to 10 .

$$
c_{\mathrm{D}}=c / \alpha^{\mathrm{D}-4},
$$

where $c$ is the observed speed of light in the $4 \mathrm{D}$ space-time, $C_{\mathrm{D}}$ is the quantized varying speed of light in space-time dimension number, $\mathrm{D}$, from 4 to 10 , and $\alpha$ is the fine structure constant for electromagnetism. In M-theory, the string requires $10 \mathrm{D}$, while the observed space-time is $4 \mathrm{D}$, so $\mathrm{D}$ is from 4 to 10 . (10D and $11 \mathrm{D}$ are equal in the speed of light.) Each dimensional space-time has a specific speed of light. (Since from the beginning of our observable universe, the space-time dimension has always been four, there is no observable varying speed of light in our observable universe.) The speed of light increases with the increasing space-time dimension number $\mathrm{D}$.

In special relativity, $E=M_{0} c^{2}$ modified by Equation (2) is expressed as

$$
E=M_{0} \cdot\left(c^{2} / \alpha^{2(\mathrm{D}-4)}\right)
$$




$$
=\left(M_{0} / \alpha^{2(\mathrm{~d}-4)}\right) \cdot c^{2} .
$$

Equation (3a) means that a particle in the D dimensional space-time can have the superluminal speed $c / \alpha^{\mathrm{D}-4}$, which is higher than the observed speed of light $c$, and has the rest mass $\mathrm{M}_{0}$. Equation (3b) means that the same particle in the 4D space-time with the observed speed of light acquires $M_{0} / \alpha^{2(d-4)}$ as the rest mass, where $\mathrm{d}=\mathrm{D}$. D from 4 to 10 in Equation (3a) is the space-time dimension number defining the varying speed of light. In Equation (3b), d from 4 to 10 is "mass dimension number" defining varying mass. For example, for $\mathrm{D}=10$, Equation (3a) shows a superluminal particle in eleven-dimensional space-time, while Equation (3b) shows that the speed of light of the same particle is the observed speed of light with the 4D space-time, and the mass dimension is eleven. In other words, 10D space-time can transform into 4D space-time with 10d mass dimension. 10D4d in Equation (3a) becomes 4D10d in Equation (3b) through QVSL. QVSL in terms of varying space-time dimension number, D, brings about varying mass in terms of varying mass dimension number, d. Mass dimension is also proposed in the Wesson's Space-Time-Matter (STM) theory as the matter dimension to account for the extra space dimensions [17].

The QVSL transformation transforms both space-time dimension number and mass dimension number. In the QVSL transformation, the decrease in the speed of light leads to the decrease in space-time dimension number and the increase of mass in terms of increasing mass dimension number from 4 to 10 ,

$$
\begin{gathered}
c_{\mathrm{D}}=c_{\mathrm{D}-n} / \alpha^{2 n}, \\
M_{0, \mathrm{D}, \mathrm{d}}=M_{0, \mathrm{D}-n, \mathrm{~d}+n} \alpha^{2 n}, \\
\mathrm{D}, \mathrm{d} \stackrel{\mathrm{QVSL}}{\longrightarrow}(\mathrm{D} \mp n),(\mathrm{d} \pm n)
\end{gathered}
$$

where $\mathrm{D}$ is the space-time dimension number from 4 to 10 and $\mathrm{d}$ is the mass dimension number from 4 to 10 . For example, in the QVSL transformation, a particle with 10D4d is transformed to a particle with 4D10d. In terms of rest mass, 10D space-time has 4d with the lowest rest mass, and 4D space-time has 10d with the highest rest mass.

Rest mass decreases with increasing space-time dimension number. The decrease in rest mass means the increase in vacuum energy $\left(E_{\text {vacuum }}=E-M_{0, \mathrm{D}} C^{2}\right)$, so vacuum energy increases with increasing space-time dimension number. The vacuum energy of $4 \mathrm{D}$ particle is zero. $11 \mathrm{D}$ membrane and $10 \mathrm{D}$ string are equal in the speed of light, rest mass, and vacuum energy. Since the speed of light for $>4 \mathrm{D}$ particle is greater than the speed of light for $4 \mathrm{D}$ particle, the observation of $>4 \mathrm{D}$ superluminal particles by 4D particles violates casualty. Thus, $>4 \mathrm{D}$ particles are hidden particles with respect to 4D particles. Particles with different space-time dimensions are transparent and oblivious to one another, and separate from one another if possible.

In the reversible M-theory, there are two different reversible oscillations: the oscillation between 11D and 10D and the oscillation between 10D and 4D. The reversible oscillation between 11D membrane and 10D string is described in Section 4.The transformation during the oscillation between 10D particle and 4D involves the stepwise two-step transformation: the QVSL transformation and the varying supersymmetry transformation from 10D4d to 4D4d. The QVSL transformation involves the transformation of space-time dimension, D whose mass increases with decreasing $\mathrm{D}$ for the decrease in vacuum energy. The varying supersymmetry transformation involves the transformation of the mass dimension number, $\mathrm{d}$ whose mass decreases with decreasing $\mathrm{d}$ for the fractionalization of particle, as follows.

stepwise two-step varying transformation

(1) $\mathrm{D}, \mathrm{d} \stackrel{\mathrm{QVSL}}{\longleftrightarrow}(\mathrm{D} \mp 1),(\mathrm{d} \pm 1)$

(2) $\mathrm{D}, \mathrm{d} \stackrel{\substack{\text { varying } \\ \text { supersymmetry }}}{\longrightarrow} \mathrm{D},(\mathrm{d} \pm 1)$

The repetitive stepwise two-step transformation between 10D4d and 4D4d as follows.

$$
\text { 10D4d } \leftrightarrow \text { 9D5d } \leftrightarrow \text { 9D4d } \leftrightarrow \text { 8D5d } \leftrightarrow \cdots \leftrightarrow \text { 4D5d } \leftrightarrow \text { 4D4d }
$$

In this two-step transformation, the transformation from 10D4d to 9D5d involves the QVSL transformation as in Equation (4c). Calculated from Equation (4b), the mass of 9D5d is $1 / \alpha^{2} \approx 137^{2}$ times of the mass of 10D4d. The transformation of 9D5d to 9D4d involves the varying supersymmetry transformation. In the normal supersymmetry transformation, the repeated application of the fermion-boson transformation carries over a boson (or fermion) from one point to the same boson (or fermion) at another point at the same mass. In the "varying su- 
persymmetry transformation”, the repeated application of the fermion-boson transformation carries over a boson from one point to the boson at another point at different mass dimension number in the same space-time number. The repeated varying supersymmetry transformation carries over a boson $B_{d}$ into a fermion $F_{d}$ and a fermion $F_{d}$ to a boson $\mathrm{B}_{\mathrm{d}-1}$, which can be expressed as follows

$$
\begin{gathered}
M_{\mathrm{d}, \mathrm{F}}=M_{\mathrm{d}, \mathrm{B}} \alpha_{\mathrm{d}, \mathrm{B}}, \\
M_{\mathrm{d}-1, \mathrm{~B}}=M_{\mathrm{d}, \mathrm{F}} \alpha_{\mathrm{d}, \mathrm{F}},
\end{gathered}
$$

where $M_{\mathrm{d}, \mathrm{B}}$ and $M_{\mathrm{d}, \mathrm{F}}$ are the masses for a boson and a fermion, respectively, $\mathrm{d}$ is the mass dimension number, and $\alpha_{\mathrm{d}, \mathrm{B}}$ or $\alpha_{\mathrm{d}, \mathrm{F}}$ is the fine structure constant that is the ratio between the masses of a boson and its fermionic partner. Assuming $\alpha$ 's are the same, it can be expressed as

$$
M_{\mathrm{d}, \mathrm{B}}=M_{\mathrm{d}+1, \mathrm{~B}} \alpha_{\mathrm{d}+1}^{2} \text {. }
$$

The mass of 9D4d is $\alpha^{2} \approx(1 / 137)^{2}$ times of the mass of 9D5d through the varying supersymmetry transformation. The transformation from a higher mass dimensional particle to the adjacent lower mass dimensional particle is the fractionalization of the higher dimensional particle to the many lower dimensional particles in such way that the number of lower dimensional particles becomes

$$
N_{\mathrm{d}-1}=N_{\mathrm{d}} / \alpha^{2} \approx N_{d}(137)^{2}
$$

The fractionalization also applies to D for 10D4d to 9D4d, so

$$
N_{\text {D-1 }}=N_{\text {D }} / \alpha^{2}
$$

Since the supersymmetry transformation involves translation, this stepwise varying supersymmetry transformation leads to a translational fractionalization, resulting in the cosmic expansion. Afterward, the QVSL transformation transforms 9D4d into 8D5d with a higher mass. The two-step transformation repeats until 4D4d, and then reverses stepwise back to 10D4d for the cosmic contraction. The oscillation between 10D and 4D results in the reversible cyclic fractionalization-contraction for the reversible cyclic expansion-contraction of the universe.

There is no kinetic energy in the universe where the oscillation between 10D and 4D occurs. In the Boltzmann formula of thermodynamic, the absolute entropy $\mathrm{S}$ of an ideal gas to the quantity $\mathrm{W}$, which is the number of the arrangements of particles corresponding to a given macroscopic collection of particles:

$$
S=k_{B} \ln W
$$

where $k_{B}$ is the Boltzmann's constant. The Boltzmann formula shows the relationship between entropy and the number of ways the atoms or molecules of a thermodynamic system can be arranged. The various atoms or molecules have different positions and momenta for irreversible entropy increase, because the increase in the number of different arrangements of particles in a macroscopic collection of particles requires the movements of individual particles in a macroscopic collection of particles. (There is no entropy increase in a single microscopic particle.) Individual momenta from kinetic energy are required for irreversible entropy increase in a macroscopic collection of particles. In other words, kinetic energy transforms a macroscopic collection of particles from one way of the arrangement of particles (order) into many ways of the arrangements of particles (disorder), and the process from order to disorder is irreversible in anisolated macroscopic collection of moving particles. There is no kinetic energy in the universe where the oscillation between 10D and 4D occurs. Without kinetic energy, individual particles do not have independent individual momenta, and without individual momenta for individual particles, the oscillation does not have entropy increase in a macroscopic collection of particles.

The presence of kinetic energy in our observable universe implies that the origin of kinetic energy is outside of the universe where the oscillation occurs. As shown later, the origin of kinetic energy is in the interuniversal void outside of the universe where the oscillation occurs. As shown later, the reversibility breaking of the reversible oscillation is through the creation of kinetic energy derived from the absorption of the interuniversal void by the universe. As in our universe, the absorption of the interuniversal void forced the direct transformation from 10D4d to 4D10d as the inflation followed by the Big Bang, and created kinetic energy that provided individual momenta for individual particles to produce irreversible entropy increase in a macroscopic collection of particles, resulting in the reversibility breaking of the reversible oscillation between 10D and 4D. As shown later, the inflation-Big Bang generated baryonic matter, dark matter, and kinetic energy as follows. 
10D4d + the interuniversal void $\stackrel{\text { the inflation }}{\longrightarrow} 4 \mathrm{D} 10 \mathrm{~d} \stackrel{\text { the Big Bang }}{\longrightarrow}$

baryonic matter (4D4d) + dark matter (4D5d,4D6d,4D7d,4D8d,4D9d) + kinetic energy

\section{The Reversible Multiverse}

The multiverse has been studied extensively. For example, Brian Greene [18] described the nine types of the multiverse which produce complicated collections of universes. The reversibility theory posits a simple version of the multiverse. The posited simple multiverse is the reversible multiverse that excludes any permanently irreversible physical laws and phenomena. In the reversible multiverse, the allowed universes have to be reversible cyclic universes with permanently reversible physical laws and phenomena, resulting in only limited types of allowed universes. Temporary irreversible universes are allowed. The collision of expanding universes which have the inexhaustible resource of space-time to expand is permanently irreversible due to the impossibility to reverse the collision of expanding universes. To prevent the collision of expanding universes, every universe is surrounded by the interuniversal void that is functioned as the permanent gap among universes. The interuniversal void has zero energy, zero space-time, and zero vacuum energy, while universe has non-zero energy, the inexhaustible resource of space-time to expand, and zero or/and non-zero vacuum energy. To maintain the permanent gap among universes, the interuniversal void detaches the incoming mass-energy in the interuniversal void, so mass-energy stays permanently within universes only. Surrounded by the interuniversal void as the permanent gap among universes, an expanding universe with the inexhaustible resource of space-time expands without colliding with other universes. The detachment of the incoming mass-energy in the interuniversal void prevents the collision of expanding universes. As a result, the the properties of the interuniversal void include the detachment of mass-energy in addition to zero energy, zero space-time, and zero vacuum energy. On the other hand, the properties of universe include the attachment of mass-energy in addition to non-zero energy, the inexhaustible resource of space-time to expand, and zero or/and non-zero vacuum energy. The detachment and the attachment of mass-energy are equivalent to the repulsion and the attraction of mass-energy, respectively. In other words, the interuniversal void repulses (detaches) mass-energy, while universe attracts (attaches) massenergy.

A zero-sum energy dual universe of positive energy universe and negative energy universe can be created in the zero-energy interuniversal void, and the new dual universe is again surrounded by the interuniversal void to avoid the collision of universes. Under symmetry, the new positive energy universe and the new negative energy universe undergo mutual annihilation to reverse to the interuniversal void immediately. Under symmetry breaking with the asymmetrical absorption of the interuniversal void, our dual universe has lasted a long time without reversing immediately to the interuniversal void as described later. The precise definition of the reversibility in the reversible multiverse is the reversibility between universes and the interuniversal void. All universes are created in the interuniversal void, and all universes have to be able to reverse back to the interuniversal void. The parameters involved in the formation of the same type of universes can be different. Reversible symmetry breaking and temporary irreversibility are allowed. A dual universe can be created in the interuniversal void, and equally, the "intrauniversal void" can also be created in a universe by the absorption of the interuniversal void. The intrauniversal void can be reversed to the interuniversal void by the desorption of the intrauniversal void.

\section{Reversibility Breaking Symmetry Breaking, and the Reversible Cyclic Dual Universe}

The reversibility theory posits that temporarily irreversible entropy increase is allowed through symmetry breaking, reversibility breaking, and low entropy beginning. One example is the evolution of our universe. In our universe, the symmetry breaking is the asymmetrical absorption of the interuniversal void by the dual positive-negative energy universe. The positive energy universe absorbed the interuniversal void, while the negative universe did not. As shown later, the absorption of the interuniversal void breaks the reversibility in the reversible M-theory. As a result, the positive energy universe becomes the locally irreversible universe, while the negative energy universe without absorbing the interuniversal void is the locally reversible universe. Because the multiverse is permanently reversible, the locally irreversible positive energy universe is temporally irreversible. The reversible negative energy universe that guides the reversible process allows the dual universe as a whole to be reversible. Our dual universe is the globally reversible cyclic dual universe as shown in Equation (10) and 
Figure 1 for the evolution of our universe.

the dual positive energy-negative energy universe $\stackrel{\text { symmetry breaking-reversibility breaking }}{\longrightarrow}$

the locally irreversible positive energy universe + the locally reversible negative energy universe

$\longrightarrow$ the globally reversible cyclic dual universe

As described previously [2]-[4], the reversible cyclic universe starts in the zero-energy interuniversal void, which produces the dual universe of the positive energy 11D membrane universe and the negative energy 11D membrane universe as in Figure 1. In some dual 11D membrane universes, the 11D positive energy membrane universe and the negative energy 11D membrane universe coalesce to undergo annihilation and to return to the interuniversal void as in Figure 1. On the other hand, in some dual 11D membrane universes like our dual universe under the reversible oscillation between 11D and 10D, the positive energy 11D membrane universe and the negative energy 11D membrane universe are transformed into the positive energy 10D string universe and the negative energy 10D string universe as in Figure 1. The positive energy 11D membrane universe is transformed into the positive energy 10D string universe as in Equations (11a) and (11b).

The RS1 Membrane Transformation

$$
\begin{aligned}
2_{11} \stackrel{\text { from 11D membrane to 10D string }}{\longrightarrow} 1_{10} \text { in the } 11 \mathrm{D} \text { AdS space } & \text { (step1) } \\
2\left(1_{10}\right) \stackrel{\text { the close string vibration }}{\longrightarrow} & 1_{10} 0_{10}=1_{10} g_{e} \text { in the 11D AdS space } \\
2\left(2_{11}\right) \stackrel{\text { the closestring and the open string vibrations }}{\longleftrightarrow}\left(s 1_{10}\right) g_{e} &
\end{aligned}
$$

where $2_{11}$ is membrane (denoted as 2 ) in $11 \mathrm{D}$, $\mathrm{s}$ is the pre-strong force, $1_{10}$ is string (denoted as 1 ) in $10 \mathrm{D}, 0_{10}$ is particle (denoted as 0 ) in 10D, AdS is anti-de Sitter, and $g_{\mathrm{e}}$ is the external graviton.

As shown in Equation (11a), one of the possible membrane transformations from the 11D membrane to the 10D string is the RS1 membrane transformation [19] [20] which involves two steps. 1) The extra spatial dimension of the 11D membrane in the transformation from the 11D membrane to the 10D string becomes the spatial dimension transverse to the string brane in the bulk 11D anti-de Sitter space [19]. This transformation is derived from the transformation from membrane to string. In the transformation from the two-dimensional membrane to the one-dimensional string, the extra spatial dimension of the two-dimensional membrane on the $x-y$ plane becomes the $\mathrm{x}$-axis transverse to the one-dimensional string on the y-axis in the two-dimensional $\mathrm{x}$-y space. 2) For the RS1 membrane transformation, two string branes are combined into the combined string brane. The external 10D particles generated by the close string vibration of the combined string brane are the 10D external gravitons which form the external graviton brane as the Gravitybrane (Planck Plane) in the RS1 of the Randall-Sundrum model [19] [20]. As in the RS1 of the Randall-Sundrum model, the two branes with equal mass-energy in the 11D anti-de Sitter space are the string brane with weak gravity and the external graviton brane with strong gravity.

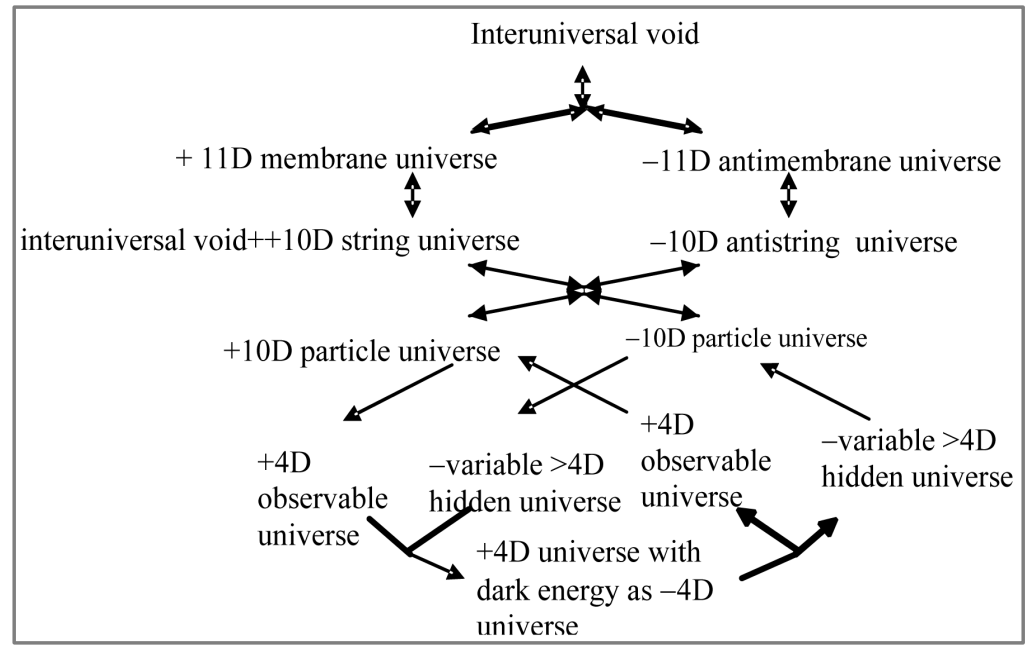

Figure 1. The globally reversible cyclic dual universe. 
The weak gravity in the string brane is the predecessor of the observed weak gravity generated during the Big Bang [2] [7]. The external graviton in the external graviton brane is the predecessor of a part of the observed dark energy [6]. The 10D string brane and the 10D external graviton brane correspond to the predecessors of the observed universe (without dark energy) and a part of observed dark energy, respectively [2] [6] [7]. The reverse transformation from 10D to 11D is the RS1 string transformation.

In Equation (11b), the 10D particles generated from the open string vibration are the 10D particles for the pre-strong force (denoted as s) which is the same for all strings without positive or negative sign. This pre-strong force is the prototype of the observed strong force generated during the Big Bang [2] [7]. The 10D particle for the pre-strong force from the open string vibration can only be in the 10D string brane. 11D membrane, 10D string, and 10D external graviton is equal in the speed of light, mass-energy, and vacuum energy.

In the negative universe through symmetry, the $11 \mathrm{D}$ anti-membrane $\left(2_{-11}\right)$ is transformed into $10 \mathrm{D}$ antistring $\left(1_{-10}\right)$ with external anti-graviton ge and the pre-strong force $s$ as in Equation (12).

$$
2\left(2_{-11}\right) \leftrightarrow\left(s 1_{-10}\right) \overline{g_{e}}
$$

The dual universe of the positive energy 10D string universe with $n$ units of $\left(1_{10}\right)_{n}$ and the negative energy 10D string universe with $\mathrm{n}$ units of $\left(1_{-10}\right)_{\mathrm{n}}$ is as follows.

$$
\left(\left(s 1_{10}\right) g_{e}\right)_{n}\left(\overline{g_{e}}\left(s 1_{-10}\right)\right)_{n}
$$

There are four equal regions: the positive energy 10D string universe, the external graviton, the external antigraviton, and the negative energy 10D antistring universe.

Some dual 10D string universesreturn to the dual 11D membrane universes under the reversible oscillation between 11D and 10D. Alternatively, under symmetry breaking as in the case of our universe, the positive energy 10D string universe absorbs the interuniversal void, while the negative energy 10D string universe does not absorb the interuniversal void as described previously [2]. (In our universe, the external graviton and the external anti-gravitonin the four regions also do not absorb the interuniversal void, so the percentage the area that does not absorb the interuniversal void is $75 \%$, three out of four regions, as the maximum percentage of dark energy described previously [6]). The interuniversal void has zero vacuum energy. The absorption of the interuniversal void by the positive energy 10D string universe forces the positive energy 10D universe with high vacuum energy to be transformed into the universe with zero vacuum energy that is the vacuum energy of the $4 \mathrm{D}$ universe. However, the transformation from $10 \mathrm{D}$ to $4 \mathrm{D}$ is not immediate, because the strings have to be $10 \mathrm{D}$, and it cannot be transformed into $4 \mathrm{D}$, therefore, strings have to be transformed into particles that allow the change of its dimension number freely to accommodate the transformation from the 10D universe to the $4 \mathrm{D}$ universe driven by the absorption of the interuniversal void. As described previously [2], the transformation of strings into particles comes from the emergence of positive charge and negative charge that allows the mutual annihilation of positively charged 10D strings and negatively charged 10D antistrings in the 10D string universes to produce positively charged 10D particles and negatively pre-charged 10D antiparticles in the 10D particle universes. The emergence of positive charge and negative charge provides the prototype of the observed electromagnetic force with chargegenerated during the Big Bang [2] [7]. The emergence of the asymmetrical dual universe provides the prototype of the observed asymmetrical weak forcegenerated during the Big Bang [2] [7]. The emergence of positive charge and negative charge and the emergence of the asymmetrical dual universe occur at the same time, so the electromagnetic force and the weak force are unified as the electroweak force [2] [7] in the Standard Model.

The transformation from the 10D4d positive energy universe with high vacuum energy to the 4D10d positive energy universe with zero vacuum energy is the inflation. At zero vacuum energy, the absorbed interuniversal void is transformed into "the 4D intrauniversal void" with zero vacuum energy and with space-time inside of the universe as described previously [3]. (The absorption of the interuniversal void by the positive energy 10D universeis in the latent stage before the 4D positive energy universe, and is in the active stage in the 4D positive energy universe.) Derived from the interuniversal void, the intrauniversal void has the same property of the detachment of mass-energy as the interuniversal void described in Section 3. Inside of the universe, the intrauniversal void with the property of the detachment of mass-energy detaches adjacent mass-energy in the universe, resulting in the conversion of rest mass (massive particles) into kinetic energy (massless particles), so the intrauniversal void is the reverse Higgs field generating massless particles starting the first step of the Big Bang in the universe with the inexhaustible resource of space-time to expand. The Higgs field inside of the universe is desorbed to become the 
external Higgs field outside of the universe. In terms of the Higgs mechanism, the reverse Higgs field derived from the interuniversal void desorbs the longitudinal component (the Higgs field) inside of the universe to become the external Higgs field outside of the universe, and generated massless particles. The equation for the first step of the Big Bang is as Equation (14)

$$
\text { interuniversal void }+ \text { Higgs field } \rightarrow \text { reverse Higgs field + external Higgs field }
$$

The second step of the Big Bang is the exchange process between the partial absorption of the Higgs bosons (the mediating particles) from the external Higgs field and the partial desorption of the reverse Higgs field inside to become the interuniversal void outside of the universe. Under symmetry breaking, the second step is the partial reversal of the first step. In terms of the Higgs mechanism, the absorbed Higgs bosons provide the longitudinal components for massless particles to form massive particles, and desorbed the reverse Higgs field inside to become the interuniversal void outside to complete the exchange process. Photon without absorbing the Higgs boson remains massless in the partial conversion, and the unabsorbed Higgs boson becomes the observed Higgs boson [8]. The equation for the second step of the Big Bang is as Equation (15).

$$
\begin{aligned}
& \stackrel{\substack{\text { reverse Higgs field + Higgs boson from the external Higgs field } \\
\text { partial }}}{\longrightarrow} \text { Higgs field + interuniversal void }
\end{aligned}
$$

In summary, the two-step process during the Big Bang included 1) the conversion of rest mass (massive particles) into kinetic energy (massless particles) by the creation of the reverse Higgs field derived from the absorption of the interuniversal void outside of the universe and 2) the partial conversion of massless particles into partial massive particles by the partial absorption of the Higgs bosons from the external Higgs field outside of the universe.

$$
\stackrel{\substack{\text { partial absorption of Higgs } \\ \text { bosons from external Higgs field }}}{\longrightarrow} \text { partial massive particles }
$$

The absorption of the interuniversal void forced the direct transformation from 10D4d to 4D10d as the inflation followed by the Big Bang, and created kinetic energy that provided individual momenta for individual particles to produce irreversible entropy increase in a macroscopic collection of particles, resulting in the reversibility breaking of the reversible oscillation between 10D and 4D. The positive energy universe becomes the locally irreversible universe. Without absorbing the interuniversal void, the coexisting negative energy universe follows the reversibility of the reversible M-theory becomes the locally reversible universe that oscillates between 10D and 4D dimension by dimension. The repetitive stepwise two-step transformation from 10D4d to 4D10d as follows.

$$
\begin{aligned}
& \text { 10D4d } \leftrightarrow \text { 9D5d } \leftrightarrow \text { 9D4d } \leftrightarrow \text { 8D5d } \leftrightarrow \cdots \leftrightarrow \text { 4D5d } \leftrightarrow \text { 4D4d } \\
& \mapsto \text { hidden negative energy universe } \quad \leftarrow \mapsto \text { dark energy } \leftarrow
\end{aligned}
$$

The negative energy universe universe consists of two periods: the hidden negative energy universe and the dark energy universe. The hidden negative energy universe composes of the $>4 \mathrm{D}$ particles. As mentioned before, particles with different space-time dimensions are transparent and oblivious to one another, and separate from one another if possible. Thus, $>4 \mathrm{D}$ particles are hidden and separated particles with respect to 4D particles in the positive energy universe (our observable universe). The hidden negative energy universe with $\mathrm{D}>4$ and the observable universe with $\mathrm{D}=4$ are the "parallel universes". The 4D particles transformed from hidden $>4 \mathrm{D}$ particles in the negative energy universe are observable dark energy for the positive energy universe, resulting in the accelerated expanding universe. Since the dark universe without kinetic energy, the presence of dark energy is not different from the presence of the cosmological constant. In terms of quintessence, such dark energy can be considered the tracking quintessence [21] from the negative energy universe with the space-time dimension number as the tracker. Observable dark energy emerged about 5 billion years ago (more precisely $4.71 \pm 0.98$ billion years ago at $\mathrm{z}=0.46 \pm 0.13$ [22]. According to the calculation in the cyclic universe [6], dark energy started in 4.28 billion years ago in agreement with the observed value. After the appearance as the dark energy, the negative energy universe will move back to $\mathrm{D}>4$ dimension by dimension, and disappear from the positive energy universe starting the contraction until the Big Crush followed by the deflation. Then, the universe will repeat the cycle starting the inflation-Big Bang or go back to the interuniversal void as in Figure 1.

The multiverse can be considered as the digital multiverse consisting of +1 for positive energy universe, -1 
for negative energy universe, and 0 for the interuniversal void. The sum in the digital multiverse is 0 , so the multiverse is the reversible digital zero-sum multiverse. During the Big Bang, the combination of the positive energy universe as 1 and the interuniversal void as 0 in the digital multiverse led to the digital space structure consisting of attachment space (the Higgs field) as 1 and detachment space (the reverse Higgs field) as 0 in the positive energy universe as in Equation (18).

$$
\begin{aligned}
& \text { positive energy universe(1)+ interuniversal void( } 0) \\
& \rightarrow \text { attachment space as Higgs field }(1)+\text { detachment space as reverse Higgs field }(0)
\end{aligned}
$$

The digital space structure is described in the previous paper [5] [14]. Derived from the space of the universe before the Big Bang, attachment space is the Higgs field, and attaches to mass-energy continuously or reversibly. Derived from the interuniversal void outside of the universe, detachment space is the reverse Higgs field, and detaches adjacent mass-energy continuously. Attachment space relates to massive particles, rest mass and reversible movement, while detachment space relates to massless particles and kinetic energy adjacent to detachment space continuously.

The combination of $n$ units of attachment space as 1 and $n$ units of detachment space as 0 brings about three different space structures: binary partition space, miscible space, or binary lattice space as below.

$$
(1)_{n} \quad+\quad(0)_{n} \stackrel{\text { combination }}{\longrightarrow}(1)_{n}(0)_{n}, \quad(1+0)_{n} \quad \text {, or } \quad(10)_{n}
$$

attachment space detachment space binary partition space, miscible space, binary lattice space

Binary partition space, $(1)_{\mathrm{n}}(0)_{\mathrm{n}}$, consists of two separated continuous phases of multiple quantized units of attachment space and detachment space. In miscible space, $(1+0)_{n}$, attachment space is miscible to detachment space, and there is no separation of attachment space and detachment space. Binary lattice space, $(10)_{n}$, consists of repetitive units of alternative attachment space and detachment space.

Binary partition space is the space for wavefunction in quantum mechanics. In wavefunction,

$$
|\Psi\rangle=\sum_{i=1}^{n} c_{i}\left|\phi_{i}\right\rangle
$$

Each basis element, $\left|\phi_{i}\right\rangle$, has both attachment space and detachment space as binary partition space. Neither attachment space nor detachment space is zero in binary partition space for a basic element. The measurement in the uncertainty principle in quantum mechanics is essentially the measurement of attachment space size and momentum from the detachment space in binary partition space: large momentum from detachment space has small non-zero attachment space size, while large attachment space size has low non-zero momentum from detachment space. In binary partition space, an entity is both in constant motion as wave for detachment space and in stationary state as a particle for attachment space, resulting in the wave-particle duality.

In binary partition space, for every detachment space, there is its corresponding adjacent attachment space. Thus, no part of the mass-energy can be irreversibly separated from binary partition space, and no part of a different mass-energy can be incorporated in binary partition space. Binary partition space represents coherence as wavefunction. Binary partition space is for coherent system. Any destruction of the coherence by the addition of a different mass-energy to the mass-energy causes the collapse of binary partition space into miscible space. The collapse is a phase transition from binary partition space to miscible space.

$$
(0)_{n}(1)_{n} \stackrel{\text { collapse }}{\longrightarrow}(0+1)_{n}
$$

binary partition space miscible space

The information in miscible space is contributed by the miscible combination of both attachment space and detachment space, so information can no longer be non-localized. Any value in miscible space is definite. All observations in terms of measurements bring about the collapse of wavefunction, resulting in miscible space that leads to eigenvalue as definite quantized value. Such collapse corresponds to the appearance of eigenvalue, E, by a measurement operator, $\mathrm{H}$, on a wavefunction, $\Psi$.

$$
H \Psi=E \Psi
$$

In miscible space, attachment space is miscible to detachment space, and there is no separation of attachment space and detachment space. In miscible space, attachment space contributes zero speed, while detachment space 
contributes the speed of light. For a moving massive particle consisting of a rest massive part and a massless part, the massive part with rest mass, $m_{0}$, is in attachment space, and the massless part with kinetic energy, $K$, is adjacent to detachment space. The combination of the massive part in attachment space and massless part in detachment leads to the propagation speed in between zero and the speed of light. To maintain the speed of light constant for a moving particle, the time $(\mathrm{t})$ in moving particle has to be dilated, and the length $(\mathrm{L})$ has to be contracted relative to the rest frame.

$$
\begin{aligned}
& t=t_{0} / \sqrt{1-v^{2} / c^{2}}=t_{0} \gamma, \\
& L=L_{0} / \gamma, \\
& E=K+m_{0} c^{2}=\gamma m_{0} c^{2}
\end{aligned}
$$

where $\gamma=1 /\left(1-v^{2} / c^{2}\right)^{1 / 2}$ is the Lorentz factor for time dilation, and length contraction, $E$ is the total energy, and $K$ is the kinetic energy.

Bounias and Krasnoholovets [23] propose that the reduction of dimension can be done by slicing dimension, such as slicing 3 space dimension object (block) into infinite units of 2 space dimension objects (sheets). The positive energy 10D4d particle universe as our observable universe with high vacuum energy was transformed into the 4D10d universe with zero vacuum energy at once, resulting in the inflation. During the Big Bang following the inflation, the 10d (mass dimension) particle in attachment space denoted as 1 was sliced by detachment space denoted as 0 . For example, the slicing of $10 \mathrm{~d}$ particle into $4 \mathrm{~d}$ particle is as follows.

$$
\left.\begin{array}{ccc}
1_{10} \stackrel{\text { slicing }}{\longrightarrow} 1_{4} & \sum_{\mathrm{d}=5}^{10}\left(0_{4} 1_{4}\right.
\end{array}\right)_{n, \mathrm{~d}}
$$

where $1_{10}$ is $10 \mathrm{~d}$ particle, $1_{4}$ is $4 \mathrm{~d}$ particle, $\mathrm{d}$ is the mass dimension number of the dimension to be sliced, $\mathrm{n}$ as the number of slices for each dimension, and $\left(0_{4} 1_{4}\right)_{n}$ is binary lattice space with repetitive units of alternative $4 \mathrm{~d}$ attachment space and $4 \mathrm{~d}$ detachment space. For $4 \mathrm{~d}$ particle starting from $10 \mathrm{~d}$ particle, the mass dimension number of the dimension to be sliced is from $d=5$ to $d=10$. Each mass dimension is sliced into infinite quantized units $(n=\infty)$ of binary lattice space, $\left(0_{4} 1_{4}\right)_{\infty}$. For $4 d$ particle, the $4 d$ core particle is surrounded by 6 types (from $d=5$ to $d=10$ ) of infinite quantized units of binary lattice space. Such infinite quantized units of binary lattice space represent the infinite units $(n=\infty)$ of separate virtual orbitals in a gauge force field, while the dimension to be sliced is "dimensional orbital" (DO), representing a type of gauge force field. The mass-energy in each dimensional orbital increases with the number of dimension number, and the lowest dimension orbital with $d=5$ has the lowest mass-energy [7] [11]. 10d particle was sliced into six different particles: 9d, 8d, 7d, 6d, 5d, and 4d equally by mass. Baryonic matter is $4 \mathrm{~d}$, while dark matter consists of the other five types of particles $(9 \mathrm{~d}, 8 \mathrm{~d}, 7 \mathrm{~d}$, 6d, and 5d).

$$
\begin{aligned}
& \text { 10D4d + the interuniversal void } \stackrel{\text { the inflation }}{\longrightarrow} \text { 4D10d } \stackrel{\text { the Big Bang }}{\longrightarrow} \\
& \text { baryonic matter }(4 \mathrm{D} 4 \mathrm{~d})+\text { dark matter }(4 \mathrm{D} 5 \mathrm{~d}, \text { 4D6d, 4D7d, 4D8d, 4D9d) + kinetic energy }
\end{aligned}
$$

The mass ratio of dark matter to baryonic matter is 5 to 1 . At $72.8 \%$ dark energy, the calculated values for baryonic matter and dark matter (with the $1: 5$ ratio) are $4.53 \%(=(100-72.8) / 6)$ and $22.7 \%(=4.53 \times 5)$, respectively, in excellent agreement with observed $4.56 \%$ and $22.7 \%$, respectively [24]. The dimensional orbitals of baryonic matter provide the base for the periodic table of elementary particles to calculate accurately the masses of all elementary particles, including quarks, leptons, gauge bosons, the Higgs boson, and the knees-ankles-toe in cosmic rays [7]-[9]. The calculated masses of all elementary particles are in good agreement with the observed values.

The lowest dimensional orbital is for electromagnetism. Baryonic matter with maximum number of gauge force fields (dimensional orbitals) is the only one with the lowest dimensional orbital for electromagnetism. With higher dimensional orbitals, dark matter does not have this lowest dimensional orbital [5] [6]. Without electromagnetism, dark matter cannot emit light, and is incompatible to baryonic matter with electromagnetism, like the incompatibility between oil and water. Derived from the incompatibility between dark matter and baryonic matter, the modified interfacial gravity (MIG) between homogeneous baryonic matter region and homogeneous dark matter region to separate baryonic matter region and dark matter region explains galaxy evolution and unifies the 
CDM (Cold Dark Matter) model, MOG (Modified Gravity), and MOND (Modified Newtonian Dynamics) [10] [11]. The digital space structure based on the combination of binary partition space and binary lattice space explains superconductivity [12] and superstar without singularity to replace black hole with singularity [13] [14]. Singularity is permanently irreversible by losing information permanently, forbidden in the reversible multiverse.

\section{Temporary Irreversible Entropy Increase}

In the reversibility theory, all physical laws and phenomena are permanently reversible. The allowed temporary irreversible universes with irreversible entropy increase must be able to return to the reversible universes with permanently reversible physical laws and phenomena. The requirements for temporary irreversible entropy increase are 1) reversibility breaking, 2) symmetry breaking, and 3) low entropy beginning.

The allowed reversibility breaking of the reversible oscillation between 10D and 4D is by kinetic energy derived from the absorption of the interuniversal void by a universe, The absorption of the interuniversal void forces the direct transformation from 10D to $4 \mathrm{D}$ as the inflation followed by the Big Bang, and creates kinetic energy that provides individual momenta for individual particles to produce irreversible entropy increase in a macroscopic collection of particles, resulting in the reversibility breaking of the reversible oscillation between 10D and 4D. Such reversibility breaking can occur only through symmetry breaking where one universe becomes irreversible, while the coexisting universe in the dual universe remains reversible to serve as a guide for the reversible dual universe as a whole. If both universes in the dual universe absorb the interuniversal void, both universes are irreversible and the dual universe will become permanently irreversible which is forbidden in the reversibility theory.

The reversibility breaking that starts the beginning of the irreversible entropy increase is caused by the absorption of the interuniversal void. In the reversible cyclic dual universe, the absorption of the interuniversal void is the exact time-reversed version of the desorption of the intrauniversal void. To have exactly reversible absorption-desorption of the interuniversal void for the reversible dual universe, the absorption and desorption have to be uniform. The space of the universe where the absorption-desorption occurs has to be small enough for the uniform absorption-desorption. The 10D4d universe with a high vacuum energy has a small space. During the inflation, the 10D4d universe was transformed into the 4D10d universe. According to Equation (4b), $\mathrm{M}_{10 \mathrm{D} 4 \mathrm{~d}}=$ $\mathrm{M}_{4 \mathrm{D} 10 \mathrm{~d}} \alpha^{12} \approx \mathrm{M}_{4 \mathrm{D} 10 \mathrm{~d}} / 137^{12}=\mathrm{M}_{4 \mathrm{D} 10 \mathrm{~d}} /\left(4.4 \times 10^{25}\right)$. The 10D4d universe where the interuniversal void was absorbed was much smaller than the 4D10d universe. Kinetic energy emerged in the Big Bang caused the further expansion of the universe. The reversible absorption-desorption of the interuniversal void forbids any absorption of the interuniversal void in the large universe which cannot be uniform. A small space results in low entropy beginning such as in the inflation-Big Bang for the beginning of the irreversible entropy increase.

\section{Summary}

In the posited reversibility theory, 1) all physical laws and phenomena are permanently reversible, 2) all physical laws are derived from the reversible M-theory (extension of string theory) in the reversible multiverse, and 3) temporary irreversible entropy increase is allowed through reversibility breaking, symmetry breaking, and low entropy beginning. Dealing with all physical laws and phenomena, the reversibility theory is the theory of everything, including thermodynamic, cosmology, the composition (baryonic matter, dark matter, and dark energy) in the universe, the periodic table of all elementary particles, the galaxy evolution, superconductivity, and black hole. In the reversible M-theory, space-time dimension number oscillates between 11D (space-time dimension) and 10D and between 10D and 4D. The reversible M-theory includes 11D membrane, 10D string, and variable D particle. Space-time dimension number between 10 and 4 decreases with decreasing speed of light, decreasing vacuum energy, and increasing rest mass. In our dual universe of positive-negative energy universe, the reversible oscillation between 10D with and 4D in the negative energy universe without kinetic energy is the reversible cyclic fractionalization-condensation for the reversible cyclic expansion-contraction of the universe. Without kinetic energy, the individual particles have no individual momenta for entropy increase. The negative energy universe is the locally reversible universe for dark energy. In the positive energy universe where we live, the absorption of the interuniversal void forced the direct transformation from 10D to 4D as the inflation followed by the Big Bang, and created kinetic energy that provided individual momenta for individual particles to produce irreversible entropy increase, resulting in the reversibility breaking of the reversible oscillation. The positive energy universe is the locally irreversible universe. The dual universe as a whole is reversible guided by the 
reversible negative energy universe. As a result, the irreversible positive energy universe with the irreversible entropy increase is temporary, and will disappear.

\section{References}

[1] Carroll, S. (2010) From Eternity to Here: The Quest for the Ultimate Theory of Time. Plume, New York.

[2] Chung, D. (2015) The Reversible Cyclic Universe in the Reversible Multiverse and the Reversible String Theory. Journal of Modern Physics, 6, 1249-1260. http://dx.doi.org/10.4236/jmp.2015.69130

[3] Chung, D. (2015) The Big Bang Started by the Creation of the Reverse Higgs Field. Journal of Modern Physics, 6 , 1189-1194. http://dx.doi.org/10.4236/jmp.2015.69123

[4] Chung, D. and Krasnoholovets, V. (2013) The Light-Dark Dual Universe for the Big Bang and Dark Energy. Journal of Modern Physics, 4, 77-84.

[5] Chung, D. and Krasnoholovets, V. (2013) The Space Structure, Force Fields, and Dark Matter. Journal of Modern Physics, 4, 27-31.

[6] Chung, D. (2014) String Theory with Oscillating Space-Time Dimension Number. Journal of Modern Physics, 5, 464472. http://dx.doi.org/10.4236/jmp.2014.56056

[7] Chung, D. (2014) The Periodic Table of Elementary Particles Based on String Theory. Journal of Modern Physics, 5, 1234-1243. http://dx.doi.org/10.4236/jmp.2014.514123

[8] Chung, D. and Hefferlinm, R. (2013) The Higgs Boson in the Periodic System of Elementary Particles. Journal of Modern Physics, 4, 21-26.

[9] Chung, D. (2014) The Knees-Ankles-Toe in Cosmic Rays and the Periodic Table of Elementary Particles. Journal of Modern Physics, 5, 1467-1472. http://dx.doi.org/10.4236/jmp.2014.515148

[10] Chung, D. (2014) Galaxy Evolution by the Incompatibility between Dark Matter and Baryonic Matter. International Journal of Astronomy and Astrophysics, 4, 374-383. http://dx.doi.org/10.4236/ijaa.2014.42032

[11] Chung, D. (2015) The Modified Interfacial Gravity: Unifying CDM, MOG, and MOND. Global Journal of Science Frontier Research A, 15, 119-125.

[12] Chung, D. (2015) The Basic Cause of Superconductivity. Journal of Modern Physics, 6, 26-36. http://dx.doi.org/10.4236/jmp.2015.61005

[13] Chung, D. and Krasnoholovets, V. (2013) Singularity-Free Superstar as an Alternative to Black Hole and Gravastar. Journal of Modern Physics, 4, 1-6.

[14] Chung, D. (2014) The Digital Space Structure, Superconductor, and Superstar. Global Journal of Science Frontier Research $A, 14,1-8$.

[15] Albrecht, A. and Magueijo, J. (1999) A Time Varying Speed of Light as a Solution to Cosmological Puzzles. Physics Review D, 59, Article ID: 043516.

[16] Barrow, J.D. (2003) Unusual Features of Varying Speed of Light Cosmologies. Physics Letters B, 564, 1-7. http://dx.doi.org/10.1016/S0370-2693(03)00573-2

[17] Wesson, P.S. (1999) Space-Time-Matter: Modern Kaluza-Klein Theory. World Scientific Publishing Company, Singapore.

[18] Greene, B. (2011) The Hidden Reality: Parallel Universes and the Deep Laws of the Cosmos. Alfred A. Knopf, New York.

[19] Randall, L. (2005) Warped Passages: Unraveling the Mysteries of the Universe's Hidden Dimensions. Harper Collins, New York.

[20] Randall, L. and Sundrum, R. (1999) A Large Mass Hierarchy from a Small Extra Dimension. Physics Review Letter, 83, 3370-3373. http://dx.doi.org/10.1103/PhysRevLett.83.3370

[21] Padmanabhan, T. (2003) Cosmological Constant-The weight of the Vacuum. Physical Report, 380, $235-320$. http://dx.doi.org/10.1016/S0370-1573(03)00120-0

[22] Riess, A.G., Strolger, L.-G., Tonry, J., Casertano, S., Ferguson, H.C., Mobasher, B., et al. (2004) Type I $\mathrm{I}_{\mathrm{a}}$ Supernova Discoveries at $z>1$ from the Hubble Space Telescope: Evidence for Past Deceleration and Constraints on Dark Energy Evolution. Astrophysical Journal, 607, 665-687. http://dx.doi.org/10.1086/383612

[23] Bounias, M. and Krasnoholovets, V. (2003) Scanning the Structure of Ill-Known Spaces: Part3. Distribution of Topological Structures at Elementary and Cosmic Scales. The International Journal of Systems and Cybernetics, 32, $1005-1020$.

[24] Jarosik, N., Bennett, C.L., Dunkley, J., Gold, B., Greason, M.R., Halpern, M., et al. (2011) Seven-Year Wilkinson Microwave Anisotropy Probe (Wmap ${ }^{*}$ ) Observations: Sky Maps, Systematic Errors, and Basic Results. The Astrophysical Journal Supplement Series, 192, 14. http://dx.doi.org/10.1088/0067-0049/192/2/14 